\title{
Innovative staffing in emergency departments: the role of midlevel providers
}

\author{
Kevin Klauer, DO, EJD
}

Historically, emergency department (ED) staffing was similar in the United States and Canada; however, during the past decade, economic pressures resulting from reduced reimbursement, uncompensated care, and increased expense have prompted US hospitals to explore and adopt more cost-effective staffing alternatives. Projected physician shortages are compounding the need to develop and expedite such

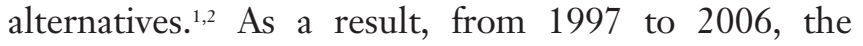
proportion of US EDs that use midlevel providers has risen from 28.3 to $77.2 \% .^{3}$ Although more prevalent in the United States, expanded staffing models are also appearing in Canada. A 2009 study in six Ontario EDs found that overall length of stay was reduced by $30.3 \%$ and $48.8 \%$ with the addition of physician assistants (PAs) and nurse practitioners (NPs), respectively. In addition, left without being treated rates fell by $44 \%$ and $71 \%$, respectively. ${ }^{4}$ Such data show promise for improving ED operational efficiency and enhancing revenue by reducing left without being seen rates, regardless of the country in which they may be located.

Unfortunately, one size does not fit all; thus, several staffing models and many variations on those models have been and are currently being used. The intention of this article is to discuss current US ED staffing models, the components of those models, and how such components can be incorporated into ED operations and workflow. These models could serve as a template for the development of alternative staffing models in Canadian EDs.

\section{A HISTORICAL PERSPECTIVE ON US ED STAFFING}

Until recently, US EDs used a traditional staffing model with physicians as the primary and often sole medical provider. Nursing functions in support of this physician-centric model were performed by registered nurses and supplemented by licensed practical nurses. Lower-level tasks that do not require significant training, certification, or licensure were often delegated to "techs."

Although projections have varied over the past decade, most sources agree that the US health care system will face a substantial physician shortage, with deficits of 45,400 primary care physicians and 46,100 specialists predicted by $2020 .{ }^{5}$ It is intuitive that these shortfalls will compromise access to primary care and increase use of EDs for urgent care and even primary care needs. A percentage of specialist shortages will be residency-trained emergency physicians; hence, ED capacity will likely be compromised at the same time there is increasing demand on EDs. It is also worthy of mention that, despite recent efforts to expand the number of graduate medical education slots in emergency medicine, new physician graduates are consumed largely by urban EDs; hence, staffing of rural departments with emergency physicians, let alone residency-trained emergency physicians, remains a major concern. ${ }^{6,7}$

In response to projected physician shortages, unmet rural ED needs, and growing economic pressure to reduce $\mathrm{ED}$ operational expense, many health systems are exploring models that reduce physician staffing by adding lower cost alternatives (i.e., PAs and advanced practice nurses). This evolution toward midlevel providers functioning autonomously or under supervision has proceeded in highly variable fashion. Although variability may suggest the need for standardization, the diversity of staffing models that has

From Emergency Medicine Physicians, Canton, OH, and Michigan State University College of Osteopathic Medicine, East Lansing, MI.

Correspondence to: Dr. Kevin Klauer, Emergency Medicine Physicians, 4281 Glenmoor Road NW, Canton, OH 44718; kklauer@emp.com.

This article has been peer reviewed. 
appeared suggests that at least one model should be applicable to any ED environment. The fundamental similarity of emergency medicine in Canada and the United States suggests that these new staffing models will likely be applicable in Canada as well.

\section{MIDLEVEL PROVIDERS: AN OVERVIEW}

The term midlevel provider (MLP) is commonly accepted in the United States; however, as PA and NP training standards expand to master's and even doctoral degrees and their role in the health care system expand, this term is no longer universally embraced, and in some settings, the term advanced practice provider has been adopted.

In the case of PAs, minimum requirements to obtain Medicare billing privileges include state licensure to practice as a PA and either graduation from an accredited PA program or having passed the national certification examination administered by the National Commission on Certification of Physician Assistants (for those who obtained training prior to 1994, by programs not accredited by the Accreditation Review Commission on Education for the Physician Assistant [ARC-PA] $){ }^{8}$ State licensure and certification are the key elements, but criteria vary from state to state. Master's training is preferred and, in fact, required in some states; however, many require only 2 years of college education, and some require a bachelor's degree (most PA applicants possess a bachelor's degree prior to matriculation). ${ }^{9}$ In addition to the aforementioned educational requirements, completion of an accredited PA program and successful completion of the national certifying examination are required for state licensure/certification (Table 1).

Advanced practice nurses (e.g., NPs) have similar requirements. For Medicare billing participation under the Centers for Medicare \& Medicaid Services (CMS), an NP must be a registered professional nurse in the relevant state and be certified by a national NP certifying body (Table 2). In addition, NPs qualifying after January 1, 2003, require a master's or doctoral degree in nursing practice. ${ }^{8}$

Controversy exists regarding use of the term doctor by advanced practice nurses who have received doctoral degrees. Many feel that they should be able to use the title as they have rightfully earned it; however, others, including some state legislatures, have regulated against this because it is misleading to

\section{Table 1. Minimum requirements for PA certification}

Successful completion of an accredited 27-month PA program

Pass the PA National Certifying Examination (PANCE)

Maintenance of Certification

100 hours of CME every 2 yr

Recertification examination every 6 yr

PANCE, National Commission on Certification of Physician

Assistants (NCCPA)

$\mathrm{CME}=$ continuing medical education; $\mathrm{PA}=$ physician assistant

patients. The primary concern is that, within a health care setting, when providers introduce themselves as "doctor," most patients believe they are being cared for by a physician who has graduated from an accredited medical school.

\section{SUPERVISORY REQUIREMENTS FOR MIDLEVEL PROVIDERS}

Level of supervision should be discussed in two separate contexts: regulatory and operational. Regulatory supervision refers to the nature of oversight mandated by regulatory bodies such as the CMS (for billing) and by state licensing agencies. Operational supervision refers to the practical oversight model developed within any given ED. Regulatory supervision is well structured, whereas operational supervision is subject to great variation from region to region and even from one ED to another.

For PAs, licensure and supervisory agreements are established and governed by the relevant state medical board. Legal requirements differ by state, but several general principles apply. PAs, by definition, are dependent practitioners, which means they must function under a supervisory agreement with a physician $^{10}$; however, the concept of supervision lacks

Table 2. State licensure requirements for nurse practitioners (Ohio Revised Code: 4723.41)

Candidates must:

1. Be a registered nurse;

2. Submit documentation satisfactory to the board that the applicant has earned a graduate degree with a major in a nursing specialty or in a related field that qualifies the applicant to sit for the certification examination of a national certifying organization listed in division (A)(3) of this section or approved by the board under section 4723.46 of the Revised Code;

3. Submit documentation satisfactory to the board of having passed the certification examinations accepted under 4723.46 .

4. Submit an affidavit with the application that verifies all educational and personal identification information. 
clear definition. To many, it implies direct involvement and real-time discussion, if not "face-to-face" patient contact and independent physician evaluation of each patient. Although some physician groups have chosen variations of this intensive supervisory model, most US states require only that the PA's work is reviewed at some point and that the physician is available for consultation should the PA need assistance. The Ohio Revised Code and the California Code of Regulations (Table 3) provide excellent examples of supervisory requirements. ${ }^{11-13}$

Most EDs have on-site physicians directly supervising or participating in PA care provision, and many physicians independently evaluate each patient to confirm the PA's findings and treatment plan. In reality, PAs tend to function much like senior-level residents, seeing a range of patients in collaboration with their physician supervisor. Although capable of seeing a broad spectrum of ED patients, day-to-day PA case mix will depend on their experience level, specific expertise, departmental care needs, and physician preferences. As is the case with residents, a physician's familiarity and comfort level with the individual PA's competence level and decisionmaking ability will determine the level of supervision applied. Of note, in some rural, remote, military, or lowacuity settings (e.g., fast track), PAs may function without on-site physician support. In addition to quality of care issues that may result from lower levels of supervision, there are also coding and billing ramifications. Shared services are billed at $100 \%$ of the physician fee schedule when physician-patient contact occurs, but only at $85 \%$ if the physician does not fulfill the "face-toface" requirement.

NPs are not governed by state medical boards but rather by the state's board of registered nursing, with regulations contained in the Nursing Practice Act. ${ }^{14}$ Historically, all states required a "collaborative agreement," which is similar to a supervisory agreement. These agreements mandated that NPs collaborate with a physician and function under established procedures and protocols. However, approximately $50 \%$ of states have removed the collaborative agreement requirement, ${ }^{15}$ giving rise to the possibility of autonomous nursing practice—something not possible for PAs.

From an operational perspective, several models of NP supervision exist. Some departments mandate direct physician supervision in all cases; others provide "direct" physician supervision only for complex or procedurally based practice. Direct supervision can involve independent physician evaluation of each patient or real-time participation through discussion only (i.e., staffing the case). Some rural and remote departments use PAs or NPs under a supervisory agreement without any direct supervision. These situations are most often related to physician shortages in specific geographic regions.

Opinions vary regarding the most appropriate supervisory model; however, based on the diversity of practice settings and the flexibility allowed by regulatory bodies, it is clear that one size does not fit all. EDs must select the model that best suits their needs, considering factors such as case mix, departmental need, staffing availability, finances, and care quality. In some cases, staffing crises due to the inability to recruit physicians necessitate a model with less supervision.

Direct supervision with independent physician evaluation of patients seen by midlevel providers is perhaps the safest approach; however, many would argue that this is overkill and that it nullifies much of the workload diversion benefit that midlevel providers bring to their departments. Nonetheless, even within a close supervisory model, midlevel providers are still able to offload physicians of burdensome and timeconsuming processes such as doing the primary history and physical examination, documenting the bulk of the ED record, and performing procedures such as suturing, splinting, laboratory and imaging follow-up, rechecks, discharge planning, and communications.

At the other extreme, many midlevel providers would prefer professional autonomy with minimal physician oversight. Autonomous practice is a consideration with NPs, even in states still requiring a collaborative agreement with a physician. In such agreements, the NP functions independently, with preestablished guidelines agreed on with the collaborating physician. However, on a day-to-day and case-by-case basis, there is no direct physician involvement. Although PAs are dependent practitioners, the supervisory agreements required by state statutes do not require direct supervision of each case or even to have a physician in the facility. Thus, from a practical standpoint, it is possible to use both PAs and NPs in a fairly autonomous practice environment. However, the complexity and risk of emergency medicine practice would suggest that autonomous practice may be less desirable, except in regions where qualified physicians are not readily available. 


\section{Table 3. State statute supervisory examples (California and Ohio)}

Supervision (CAL. CODE REGS. tit. 1681399.545)

The supervising physician may choose one or more of the following four mechanisms to provide the supervision as required by Section 1399.545 of Title 16 of the California Code of Regulations.

1. The physician sees the patients the same day that they are treated by the PA.

2. The physician reviews, signs, and dates the medical record of every patient treated by the PA within 30 days of the treatment

3. The physician adopts written protocols, which specifically guide the actions of the PA. The physician must select, review, sign, and date at least $5 \%$ of the medical records of patients treated by the PA according to those protocols within 30 days.

4. Or, in special circumstances, the physician provides supervision through another mechanism approved in advance by the PAC. Liability/Responsibility: (CAL. CODE REGS. tit. 1681399.545) Licensed physician or surgeon oversees the activities of and accepts responsibility for the medical services rendered by the PA.

Supervision (Ohio Administrative Code 4730-1-04)

A. A PA's practice shall be under the on-site supervision of the supervising physician in the following instances:

1. For practice in a health care facility's emergency department when that is a routine practice location for the supervising physician and PA;

2. During the first 500 hours of a PA's provisional period of physician-delegated prescriptive authority;

3. When on-site supervision is specified as the supervision level in a special services plan or the policies of a health care facility; and

4. When a supervising physician specifies that on-site supervision is required as a condition for the PA's performance of one or more identified services in the supervisory plan, special services plan, or policies of a health care facility.

B. A PA's practice shall be under the direct supervision of the supervising physician in the following instances:

1. When direct supervision is specified in a special services plan approved by the board; or

2. When the supervising physician specifies that direct supervision is required as a condition for the PA's performance of one or more identified services in a supervisory plan, special services plan, or the policies of a health care facility.

C. Where paragraphs $(A)$ and $(B)$ of this rule do not apply, a PA may practice under the off-site supervision of the supervising physician.

Duties of a supervising physician (Ohio Administrative Code 4730-1-03)

D. When on-site supervision or direct supervision of the physician assistant is not required under rule 4730-1-04 of the Administrative Code, the supervisory plan or special services plan approved by the board, or the policies of the health care facility in which the supervising physician and PA are practicing the supervising physician shall be continuously available for direct communication with the PA by either of the following means:

1. Being physically present at the location where the PA is practicing; or

2. Being in a location that under normal conditions is not more than 60 minutes' travel time away from the practice location of the PA and being readily available to the PA through some means of telecommunication.

\section{Table 3. Continued}

E. The supervising physician shall perform all of the following supervisory activities:

1. Personally and actively review the PA's professional activities: 2. Regularly review the condition of the patients treated by the PA

3. Regularly perform any other reviews of the PA that the supervising physician considers necessary;

4. Establish, in consultation with each PA supervised by the physician, implement, and maintain a quality assurance system in accordance with the requirements of rule 4730-1-05 of the Administrative Code;

5. Only grant prescriptive authority to a PA in compliance with the formulary adopted in rule by the board;

6. Supervise the PA's provisional period of prescriptive authority in accordance with rule 4730-2-04 of the Administrative Code; and

7. Maintain a written record of the following:

(a) Any conditions placed on a specific PA's practice in an officebased practice.

(b) Any limitations imposed in addition to any limitations applicable under the policies of a health care facility.

8. Make the written record of conditions and/or limitations required in paragraph $(E)(7)$ of this rule available on request of the board or any health care professional working with the supervising physician and PA.

$\mathrm{PA}=$ physician assistant.

Perhaps the best approach is a hybrid of these two schools of thought. In such a model, midlevel providers may be given more autonomy with lower-acuity cases but not with higher-acuity cases. The challenge is knowing which patients are truly sicker, and, unfortunately, acuity does not always find its way to the "right" ED zone. Current triage systems not infrequently allocate high-acuity patients to low-acuity treatment areas. Sore throat (epiglottitis), back pain (aortic dissection), leg pain (arterial embolism), and indigestion (myocardial infarction) patients are potential time bombs when mixed into a low-acuity, fasttrack population. If it is true that the eye does not see what the mind does not know, and that an ED physician is the best diagnostician for uncommon conditions or subtle presentations of severe illness, then the physician brings value to every case.

Replacement of physicians by midlevel providers is a growing controversy in the United States. With an evolving health care system seeking opportunities to reduce cost, the federal government has expressed interest in the use of alternative staffing models, particularly in light of anticipated physician shortages.? Compensation for a PA or NP is substantially lower than for an emergency physician. Based on a recent salary survey, average ED NP and PA compensation in 
the United States is $\$ 103,722$ and $\$ 101,688$, respectively. ${ }^{16}$ The possibility of hiring two or three midlevel providers for the same cost as one physician (depending on local and regional physician salaries) is intriguing to many health care organizations. Assuming similar productivity and quality, midlevel provider coverage is less expensive than physician coverage. In addition, the potential for improved efficiency and greater patient throughput at lower expense is a strong motivator for some and a temptation for others. Regardless of the motivation, the impact on quality of replacing physicians with midlevel providers must be factored into the decision-making process.

Coming from the perspective of dependent practitioners, PAs have not taken an organized approach to pursuing autonomous practice (i.e., to replace physicians). Conversely, some advanced practice nurses have identified this as an opportunity to expand their scope of influence in the health care delivery system and advocated strongly for NPs as a viable alternative to traditional physician-centric models of care delivery.

One recent article suggested that NP outcomes are similar to or better than those of physician comparison groups. ${ }^{17}$ This systematic review of 37 studies, published between 1990 and 2008, compared physician care to advanced practice nurse care, looking at many outcomes, including patient satisfaction, patient selfassessment of health status, functional status, blood glucose, serum lipids, blood pressure, ED visits, hospitalization, duration of ventilation, length of stay, and mortality. The measures were not specific to emergency medicine, and the populations studied ranged from nursing home patients, ambulatory care settings, and inpatient prenatal and primary care units. All but one cited outcome appeared equivalent in the physician and advanced practice nurse groups, although one measure (lipid management) was superior in the advanced practice nurse group. This evidence challenges the assumption that physician-level care is better than that provided by NPs.

Naturally, some physicians resist the introduction of NPs into their specialty, whereas others embrace it. However, system-level decisions to modify provider mix should not be based on one practitioner group's desire to expand its role-nor on another's desire to resist this. Decisions on appropriate provider mix should be based on objectively identified gaps in care delivery, as well as evaluation of operational efficiency, cost-effectiveness, quality of care, and the feasibility of preferred supervision models. During the implementation process, midlevel providers will have their own preferences in terms of practice environment, provider mix, supervision model, patient mix, and scope of practice; however, these decisions should be based on system need rather than provider preference.

\section{MEDICOLEGAL CONSIDERATIONS}

Physicians have two common misconceptions regarding collaboration with midlevel providers: first, that merely signing the chart constitutes adequate supervision and, second, that billing at $85 \%$ of the physician fee schedule (indicating no direct physician-patient contact) limits physician liability for care provided by the midlevel provider. To the surprise of many, these misconceptions lead to regulatory noncompliance and medicolegal risk. Although there are obvious differences between the Canadian and US health care systems, similar misconceptions are likely to arise as Canada develops innovative staffing models.

In the United States, signing the chart and billing at $100 \%$ of the physician fee schedule constitute fraudulent billing unless "face-to-face" time was provided and documented by the supervising physician (the CMS and most third-party payers use the shared services "face-toface" standard). ${ }^{18}$ Furthermore, in both countries, inadequate midlevel provider supervision will likely lead to substantial medicolegal risk, and billing a lower fee does not mitigate physicians' legal burden of supervision or their accountability for patient care. In the United States, physicians are still regarded as the provider with overall responsibility for all patients in the $\mathrm{ED}$, and this is unlikely to change in the foreseeable future. Consequently, if a bad outcome leads to a professional negligence claim and a lawsuit, the physician will likely be named as a codefendant for not adequately supervising midlevel care. Legal judgments may consider whether or not hospital-specific policies enabled autonomous practice or direct supervision without independent physician evaluation (i.e., discussed the case with the provider but did not independently evaluate the patient) and whether such policies limit the physician's responsibility, but these policies cannot be seen as safe harbours for medical malpractice claims. In other words, if you are physically in the ED where a midlevel provider is practicing, the plaintiff's attorneys, courts, and jurors will likely assign some degree of responsibility to the physician. 
DEFINING THE IDEAL SCOPE OF PRACTICE FOR MIDLEVEL PROVIDERS

Provider scope of practice is a topic worthy of specific treatment. What should midlevel providers be allowed to do in the ED? Among the list of standard items that most midlevel providers will be qualified and credentialed to perform are the history and physical examination, diagnosis, discharge planning, prescribing, chart completion, laceration repair, incision and drainage of subcutaneous abscesses, splinting, foreign body removal, and pelvic examinations. ${ }^{19}$ However, even with items commonly performed by PAs and NPs, differences in care are noted when compared to physicians. Steiner and colleagues attempted to define what types of patients would be suitable for autonomous NP practice by measuring their equivalency to physician care..$^{20}$ Except for follow-up care, simple lacerations, and isolated sore throats, NP care differed substantially from that of emergency physicians in their urban ED. The impact of these differences on the quality of care was not the subject of that study. However, it appears that introducing midlevel providers into the ED staffing model will likely result in changes in the care provided. Whether such changes have positively or negatively impacted the quality of care should be routinely assessed via quality assurance initiatives.

Many midlevel providers have been trained to perform and have demonstrated proficiency in other procedures as well. There is no set standard in this area, but, based on their supervisory agreements and hospital credentialing requirements, they may be credentialed to perform a variety of advanced procedures, including but not limited to central venous line placement, endotracheal intubation, joint and fracture reduction, and procedural sedation. ${ }^{19}$

Delegating advanced procedures to PAs or advanced practice nurses may save valuable physician time; however, this decision must be balanced against quality of care concerns. If both providers are equally proficient in the performance of a given procedure, then the decision becomes purely operational: Whose time is better spent performing the procedure-the midlevel provider or the physician? The answer may vary depending on the circumstances and ED needs on any given day. If the physician is more qualified to perform a procedure, and if suboptimal performance is likely to influence the patient's outcome, then the physician may have an ethical obligation to perform that procedure, despite any operational efficiency that could be gained by delegating it. There is no universally correct answer to this question; however, if advanced procedures are to be deferred to the midlevel provider, the qualifications and proficiency of the midlevel provider must be verified, quality assurance must be performed, hospital credentialing requirements must be met, and the provider's credentials (e.g., PA or NP) must be disclosed during the informed consent process.

We have largely discussed PAs and NPs in a similar light and context. Clearly, there are differences in training and supervisory requirements between the two. However, the differences in training do not result in appreciable differences in scope of practice in the ED when directly supervised. Because NPs are not dependent practitioners, they can be used in less supervised environments. What often impacts the scope of practice of any PA or NP are hospital by-laws defining the scope of practice and the previous experience of the provider. Some PAs and NPs have very little ED experience, whereas others may have extensive experience. In addition, many may have worked in certain practice environments (i.e., cardiothoracic surgery, military medicine) that have allowed them to develop a unique body of experience that should be recognized when defining their scope of practice. In a study by Doan and colleagues, pediatric emergency physicians and PAs were surveyed to determine what diagnoses might be suitable for PAs to care for in pediatric EDs. ${ }^{21}$ Thirty clinical entities, accounting for $74 \%$ of ED volume, were identified as appropriate for direct supervision, and three were selected not requiring direct supervision. Although this study did not include NPs, these data may be extrapolated to the use of NPs as they are historically used very similarly to PAs in the ED.

\section{SUMMARY}

NPs and PAs are increasingly common in US and Canadian EDs. Emergency medicine is a unique specialty with a vast knowledge base performed in a high-risk environment; therefore, supervisory models and regulatory requirements developed for lower-risk settings (e.g., primary care) and more circumscribed practices (e.g., diabetes clinic) are unlikely to be sufficient for EDs. As innovative ED staffing models 
evolve, specific roles and scopes of practice for midlevel providers should be based on an objective analysis of existing care gaps and system needs, local provider availability, operational efficiency, costeffectiveness, regulatory compliance, risk management, and quality of care. Although it is tempting to reduce cost by using PAs or NPs, de-emphasis of emergency physician involvement should proceed cautiously to avoid negatively impacting patient care.

Variability in midlevel provider use and the relative paucity of evidence describing optimal provider roles, scopes of practice, and care outcomes in different patient populations highlight the need for a measured approach, appropriate supervisory models, effective quality assurance programs, and cost-effectiveness analyses looking at both clinical and economic outcomes of this important health system evolution.

Competing interests: None declared.

Keywords: emergency department, manpower, nurse practitioner, physician assistant, staffing

\section{REFERENCES}

1. Under stress: inside the emergency department. HealthLeaders Magazine March 2003;1-9.

2. Langland-Orban B, Pracht E, Salyani S. Uncompensated care provided by emergency physicians in Florida emergency departments. Health Care Manage Rev 2005;30:315-21, doi:10.1097/00004010-200510000-00005.

3. Menchine MD, Wiechmann W, Rudkin S. Trends in midlevel provider utilization in emergency departments from 1997 to 2006. Acad Emerg Med 2009;16:963-9, doi:10.1111/j.1553-2712.2009.00521.x.

4. Ducharme J, Alder RJ, Pelletier C, et al. The impact on patient flow after the integration of nurse practitioners and physician assistants in 6 Ontario emergency departments. CFEM 2009;11:455-61.

5. Kirch DG, Henderson MK, Dill MJ. Physician workforce projections in an era of health care reform. Annu Rev Med 2012;63:435-45. [Epub 2011 Sep 1], doi:10.1146/annurevmed-050310-134634.

6. Moorhead JC, Schafermeyer RW, Rorrie CC. Key issues in emergency medicine workforce planning. Ann Emerg Med 1997;30:765-72, doi:10.1016/S0196-0644(97)70044-1.
7. Schneider SM, Gardner AF, Weiss LD, et al. The future of emergency medicine. Ann Emerg Med 2010;56:178-83, doi:10.1016/j.annemergmed.2010.04.011.

8. Centers for Medicare \& Medicaid Services (CMS). Medicare enrollment \#100-08, 2011. In: Medicare program integrity manual. Available at: http://www.cms.gov/manuals/downloads/ pim83c15.pdf (accessed February 25, 2011).

9. American Academy of Physician Assistants, Becoming a PA. Available at: http://www.aapa.org/your_pa_career/becoming a_pa.aspx (accessed September 20, 2012).

10. Department of Health and Human Services. Office of Inspector General: Medicare coverage of non-physician practitioner services. June 2001, OEI-02-00-00290.

11. CAL, CODE REGS. tit. 1681399.545. Available at: http:// www.pac.ca.gov/supervising_physicians/index.shtml (accessed September 21, 2012).

12. Obio Administrative Code 4730-1-04. Available at: http:// codes.ohio.gov/oac/4730-1 (accessed September 21, 2012).

13. Ohio Administrative Code 4730-1-03. Available at: http:// codes.ohio.gov/oac/4730-1 (accessed September 18, 2012).

14. Eibner C, Hussey PS, Ridgely MS, et al. Controlling bealth care spending in Massachusetts: an analysis of options. Santa Monica (CA): Rand Corporation; 2009. Available at: http://www. rand.org/pubs/technical_reports/TR733 (accessed September $21,2012)$

15. Flanagan L. Nurse practitioners: growing competition for family physicians? Fam Pract Manag 1998;5(9):34-43.

16. Perron-Pronsati M. National salary report 2011. ADVANCE for NPs \& PAs. February 7, 2012. Available at: http:// nurse-practitioners-and-physician assistants.advanceweb.com/ Features/Articles/National-Salary-Report-2011.aspx (accessed September 20, 2012).

17. Newhouse RP, Stanik-Hutt J, White KM, et al. Advanced practice nurse outcomes 1990-2008: a systematic review. Nurs Econ 2011;29:230-50.

18. Centers for Medicare \& Medicaid Services. Transmittal 1776. Available at: http://www.cms.hhs.gov/transmittals/ downloads/R1776B3.pdf. (accessed September 20, 2012).

19. Obio Revised Code.Title [47] XLVII occupations - professions. Chapter 4730: physician assistants. (Revised May 17, 2006).

20. Steiner IP, Blitz S, Nichols DN, et al. Introducing a nurse practitioner into an urban Canadian emergency department. CFEM 2008;10:355-63.

21. Doan Q, Sabhaney V, Kissoon N, et al. The role of physician assistants in a pediatric emergency department: a center review and survey. Pediatr Emerg Care 2012;28:783-8, doi:10.1097/PEC.0b013e3182627ce5. 